\title{
La evaluación de las curvas de producción lechera de las vacas Holstein Friesian en la estación experimental Tunshi Espoch.
}

He evaluation of the milk production curves of the Holstein Friesian cows at the Tunshi Espoch experimental station.

Segundo Manuel Shagñay Rea. ${ }^{1}$, Luis Samuel Eduardo Arias Alemán. ${ }^{2}$ \& Segundo Enrique Vaca Zambrano. ${ }^{3}$

\begin{abstract}
DOI: https://doi.org/10.33262/cienciadigital.v4i1.1070

The economic evaluation of milk production in the 2014-2018 period was carried out at the Tunshi-ESPOCH station, for which daily productive records of the cows in production were used, which were analyzed taking into account the environmental factors effects on the production of milk of the different lactations of cows. The characterization included the calculation of lactation days in weeks of adjusted production at 305 days of average lactation considered by the NRC, also based on the volume of milk production (lts) per lactation. The breastfeeding performance adjusted at 305 days was lower in first-birth cows with a production of 2675.37 / lactation, compared with cows of sixth lactation 5160.90 liters / lactation, a value that reflects the physiological and reproductive maturity of the cow, and the genetic potential demonstrated in milk production at this stage of its productive and reproductive life. The yield at peak lactation was lower in primiparous cows which fits a linear equation of fifth with (80.5 liters / week, with an average daily production of 12 liters in the first week of lactation, the same as seen influenced by intrinsic and extrinsic factors which causes them to vary as lactation passes compared to multiparous women, (108.9 liters / week, with an average daily production of 15.5 liters / day, in the first week of lactation; lactation curves are described with a fifth order equation It was determined that cows are more efficient in the sixth lactation, and that the productivity of
\end{abstract}

\footnotetext{
${ }^{1}$ Escuela Superior Politécnica de Chimborazo, Sede Morona Santiago, segundo.shagnay@espoch.edu.ec

${ }^{2}$ Escuela Superior Politécnica de Chimborazo, Sede Morona Santiago, luis.arias@espoch.edu.ec

${ }^{3}$ Escuela Superior Politécnica de Chimborazo, Sede Morona Santiago, sevacaz@espoch.edu.ec
} 
the cows is closely related to physiological maturity and milk secretion is increased as which reaches its productive maturity

Keywords: Economic Sciences, Agricultural Economics, Livestock, Evaluation, Dairy Production, Production Records, Lactation, Production Curves.

\section{Resumen}

La evaluación económica de la producción lechera en el período 2014-2018 se desarrolló en la estación Tunshi-ESPOCH, para lo cual se utilizó registros productivos diarios de las vacas en producción las cuales se analizaron tomando en consideración los efectos factores ambientales sobre la producción de leche de las diferentes lactancias de vacas. La caracterización incluyó el cálculo de los días de lactancia en semanas de producción ajustado a los 305 días de lactancia promedio considerado por la NRC, también basado en el volumen de producción de leche (lts) por lactancia. El rendimiento por lactancia ajustada a los 305 días fue menor en vacas de primer parto con una producción de 2675.37/lactancia, , en comparación con las vacas de sexta lactancia 5160.90 lts/lactancia, valor que refleja la madurez fisiológica y reproductiva de la vaca, y el potencial genético demostrado en la producción de leche a esta etapa de su vida productiva y reproductiva. El rendimiento al pico de lactancia fue menor en vacas primíparas la cual se ajusta a una ecuación lineal de quinto con (80.5 lts /semana, con un promedio de producción diaria de 12 litros en la primera semana de la lactancia, el mismo que se ve influenciado por factores intrínsecos y extrínsecos lo que hace que varíen a medida que transcurre la lactancia en comparación con las multíparas, (108.9lts/ semana, con un promedio de producción diaria de 15.5 litros/día, en la primera semana de la lactancia; las curvas de lactancia son descritas con una ecuación de quinto orden. Se determinó que las vacas son más eficientes en la sexta lactancia, , y que la productividad de las vacas está estrechamente relacionado con la madurez fisiológica y la secreción de la leche se incremente a medida que alcanza su madurez productiva.

Palabras Clave: Ciencias Económicas, Economía Agrícola, Ganadería, Evaluación, Producción Lechera, Registros de Producción, Lactancias, Curvas de Producción.

\section{Introducción}

En la actualidad la industria lechera en nuestro país busca obtener los más altos niveles de producción, lo cual se podrá lograr con la combinación correcta de los factores que inciden en ella como: La nutrición, mejoras en el manejo, instalaciones adecuadas y la selección genética; reflejándose en la curva de la producción de leche de las diferentes lactancias, y de la cual depende la vida productiva de la vaca en el hato lechero.

La producción de leche de bovino es una de las actividades pecuarias más importantes en nuestro país, ya que se realiza en la mayor parte del territorio nacional concentrando el $45 \%$ del total de la producción de leche en los medianos y pequeños productores (INEN, 2015). Por el valor de la producción, es la segunda en importancia dentro del subsector pecuario (21\%), 
después de la industria de la carne. También genera más de 200 mil empleos permanentes remunerados y apoya a 318 industrias, que generan alrededor de 500 mil empleos directos e indirectos (Sagarpa, 2009). Donde la lechería especializada aporta 85\% de la producción y la ganadería bovina de doble propósito (carne y leche) contribuye con el restante 15\% (Sagarpa, 2009). Ademas según la FAO el consumo per cápita en el Ecuador registra una cifra de 110 litros en promedio.

El comportamiento físiológico de la vaca durante su periodo de producción se puede caracterizar al analizar la curva de lactancia la que nos brinda un resumen certero de los niveles de producción de leche, determinados por la eficiencia biológica, genética de un bovino de leche en un determinado periodo y condiciones de manejo. Los modelos obtenidos a partir de los registros de producción son de mucha utilidad para los ganaderos ya que son empleados para predecir, mejorar la producción de leche en las próximas lactancias de un hato, brinda la posibilidad de tomar decisiones adecuadas del manejo y mejoramiento genético.

El Comité Internacional de Registro Animal (ICAR) establece tres métodos para el registro de producción de leche (control en ganado lechero), de acuerdo a su frecuencia cada 2, 4 o 6 semanas, al número de ordeños $1 \mathrm{X}$ (un ordeño) o $2 \mathrm{X}$ (dos ordeños) y a las instituciones o responsables de dicho registro. El método A, consiste en mediciones realizadas por un representante oficial de la organización responsable, en el método $\mathrm{B}$, todos los registros son realizados por el productor y en el método $\mathrm{C}$, las mediciones son realizadas por el productor o el administrador del aprisco, y por un representante oficial de la organización responsable

Garcia et al. (2014) mencionan que el procedimiento denominado construcción de un modelo se basa en la comparación de modelos con diferentes estructuras para las componentes aleatoria y sistemática, mediante el uso de determinadas medidas, se decide cual es el más adecuado. Aunque no existe unanimidad acerca de la manera de seleccionar el modelo óptimo.

El análisis del comportamiento de las curvas de la lactancia es importante porque permite la identificación de posibles errores de un determinado hato lechero, como la deficiente nutrición, estado sanitario y de manejo, potencial genético y las condiciones medioambientales. Permite también conocer la evolución de la producción lechera, así como sus variaciones a lo largo de la lactancia mediante el seguimiento de un animal o un gripo de ellos, estimándose de este modo el comportamiento de la producción en una o varias lactancias durante su vida productiva. Lo más relevante es que con la estimación de las curvas de lactancia, se puede identificar los animales altamente productivos de un hato, generando una herramienta útil y aplicable a la realidad de cada granja la toma de decisiones sobre el manejo productivo y reproductivo la las vacas. (León et al. 2007)

Por lo tanto la presente investigación tuvo como objetivo evaluar la curva de la producción lechera utilizando modelos matemáticos lineales en la Estación Experimental Tunshi- ESPOCH en el periodo 2014 - 2018, para que sea de utilidad al ganadero, la aplicación práctica de la curva de la lactación será determinado por el volumen de producción a 305 días, el número de lactancias y el consumo de materia seca. Para caracterizar las curvas de producción lechera es necesario conocer los modelos que mejor las definen. Esta metodología se basa en la obtención 
de una curva de lactancia estándar para grupos animales con el análisis de los registros de producción diaria, (Arango et al., 2000). El comportamiento y forma de la curva de la proporciona a los pequeños, medianos ganaderos y profesionales del área, las herramientas para analizar económicamente que tan rentable es su hato lechero y si es el caso la necesidad de hacer cambios en el manejo alimenticio, manejo y sanitario.

\section{Metodología de la investigación}

La investigación se ajusta al tipo no experimental, o cuasi experimental ya que no se aplica ningún tratamiento a su vez se enfocó en el diagnóstico mediante la recopilación de datos a través del análisis de la información obtenida de las variables del estudio, sin inmiscuirse en el acontecimiento de los resultados

\section{Métodos de Investigación.}

La presente investigación es de tipo correlacional para lo cual se utiliza el método analítico

\section{Deductivo:}

A través del método deductivo viabilizó la estructuración de los fundamentos científicos, técnicos de las estrategias de para la toma de decisiones administrativas, económicas y técnicos, y correlacionar con la información primaria obtenida del el análisis de los registros productivos.

\section{Sintético - analítico}

Con la utilización de este método se procuró reunir todos los elementos científicos, técnicos Para la toma de decisiones en base al análisis de los registros productivos

\section{Enfoque de la Investigación.}

Cuantitativo: Se miden el grado de relación entre las variables (cuantifican relaciones). Intenta predecir el valor aproximado que tendrá un grupo de fenómenos en una variable, a partir del valor que tienen en la variable relacionada. Además podemos decir que cuanto mayor número de variables estén correlacionadas o asociadas en el estudio y mayor la fuerza de correlación, más completa será la explicación.

\section{Población de estudio}

La población de estudio está compuesta por la totalidad de registros de los bovinos del programa de producción e investigación de la Estación Experimental Tinshi-Espoch.

\section{Tamaño de la muestra}

El tamaño de la muestra está dado por el total de registros de las vacas en producción del periodo 2014 - 2017 de las vacas en producción actual de la Estación Experimental Tunshi - Espoch. 


\section{Resultados Discusión.}

\section{Producción de Leche por lactancias}

Grafico 1. Producción Leche

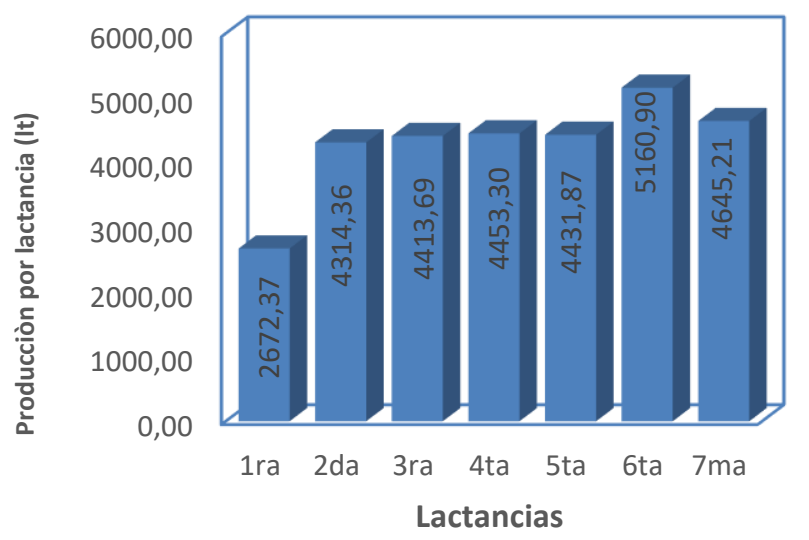

Elaborado: Segundo Shagñay. 2019.

La producción de leche de las vacas Holstein en la 2da, 3ra, 4ta, 5ta, 6ta ,7ma, lactancia fueron de: 4314.36, 4413.69, 4453.30, 4434.87, 5160.90, 4645.21 litros respectivamente, valores entre los cuales difieren significativamente $(\mathrm{P}<0,01)$ de la producción de leche en la 1ra lactancia cuyo valor fue de 2672.37 litros; esto posiblemente se deba a que las vacas en la 1ra lactancia todavía no han alcanzado su peso a la edad adulta, y recién empieza a desarrollar sus características sexuales secundarias como tamaño y capacidad de ubre, además de que los nutrientes de la alimentación están enfocándose una parte a la generación de tejido corporal para alcanzar el peso a la edad adulta. Lemus V. (2008), reporta que la producción de leche del as vacas Holstien Friesan es de 4388.7 litros/lactancia para vacas de primer parto y 5331 litros/lactancia para vacas multíparas. Fabara F. (2012) reporta una producción de 4712,55 litros/lactancia; valores superiores a las encontrados en el presente estudio, esto quizá se deba a la pureza y línea genética de las vacas, además a los sistemas de manejo de los semovientes. Lemus V. (2008) reporta un consumo de materia seca de $7120 \mathrm{Kg} / \mathrm{Ms}$ durante el ciclo productivo, valores superiores a las encontrados en el presente estudio, esto quizá se deba, al peso, a la disponibilidad de alimento y el volumen de producción láctea, a la dieta alimenticia; además a los sistemas de manejo de los semovientes. Conscientes de que este factor en vacas de razas lecheras es considerada con justificada razón como el factor fundamental para el sostenimiento de la producción de leche y a la vez como el punto crítico para lograr rentabilidad debido a que su costo representa entre el 50 y $60 \%$ del ingreso por venta de leche

\section{Modelación para la primera lactancia}

Gráfico 1-1. Comportamiento Productivo en la primera lactancia de las vacas Holstein Friesan de la Estación Experimental Tunshi - ESPOCH. 


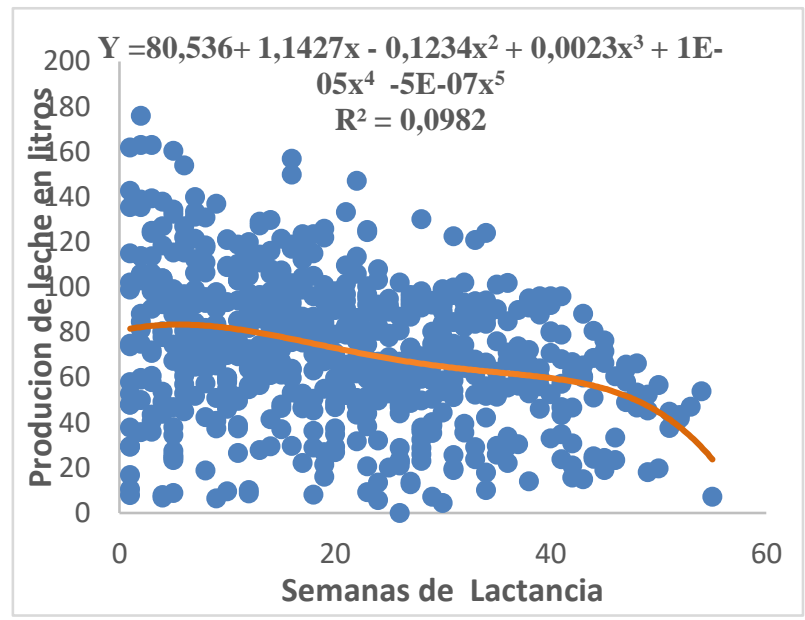

Elaborado: Segundo Shagñay. 2019.

La producción de leche generada en la primera lactancia ajustada a 305 días de las vacas Holstein Friesian responde al modelo de quinto orden $\left(\mathrm{Y}=80,536+1.1427 \mathrm{x}-0,1234 \mathrm{x}^{2}+\right.$ $\left.0,0023 x^{3}+1 E-05 x^{4}-5 E-07 x^{5}\right)$, lo que significa que por efecto del modelo la producción cuando inicia la lactancia es de 80.536 litros/ vaca/semana, y a medida que esta va transcurriendo en el tiempo esta sube o baja en función de las condiciones climáticas y nutricionales, y el estado fisiológico, concluyéndose el cese de la producción llega cuando la vaca esta próxima al siguiente parto, factores de manejo , ambientales. Además, la producción está estrechamente relacionado al desarrollo fisiológico de la glándula mamaria para la secreción de leche luego del parto, de tal manera que la vaca al llegar a la primera lactancia todavía no llega a su madurez fisiológica y por ende la glándula mamaria no completa su desarrollo, consecuentemente la produccion de leche será aproximadamente el $20 \%$ menor a su potencial.

\section{Modelación para la segunda lactancia.}

Gráfico 1-2. Comportamiento Productivo en la segunda lactancia de las vacas Holstein Friesan de la Estación Experimental Tunshi - ESPOCH.

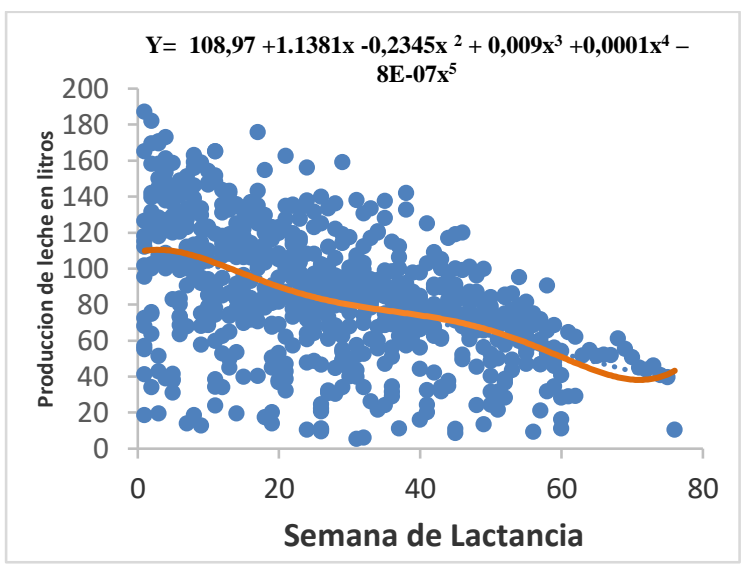

Elaborado: Segundo Shagñay. 2019. 
La producción de leche generada en la segunda lactancia ajustada a 305 días de las vacas Holstein Friesian responde al modelo de quinto orden $\left(\mathrm{Y}=108,97+1.1381 \mathrm{x}-0,2345 \mathrm{x}^{2}+\right.$ $\left.0,009 x^{3}+0,0001 x^{4}-8 E-07 x^{5}\right)$, lo que significa que por efecto del modelo en la producción cuando inicia la lactancia es de 108,97 litros/vaca/semana, y a medida que esta va transcurriendo en el tiempo esta sube con relación a la 1ra lactancia, en función del peso, el desarrollo de su habilidad reproductiva y productiva, alimentación, la fisiología del proceso de síntesis de la secreción de leche en la glándula manaría que ciertos caso ya llega a producir el nivel máximo de su potencial; el cese de la producción llega cuando la vaca esta próxima al siguiente parto, factores de manejo.

\section{Modelación para le tercera lactancia}

Gráfico 1-3. Comportamiento Productivo en la tercera lactancia de las vacas Holstein Friesan de la Estación Experimental Tunshi - ESPOCH.

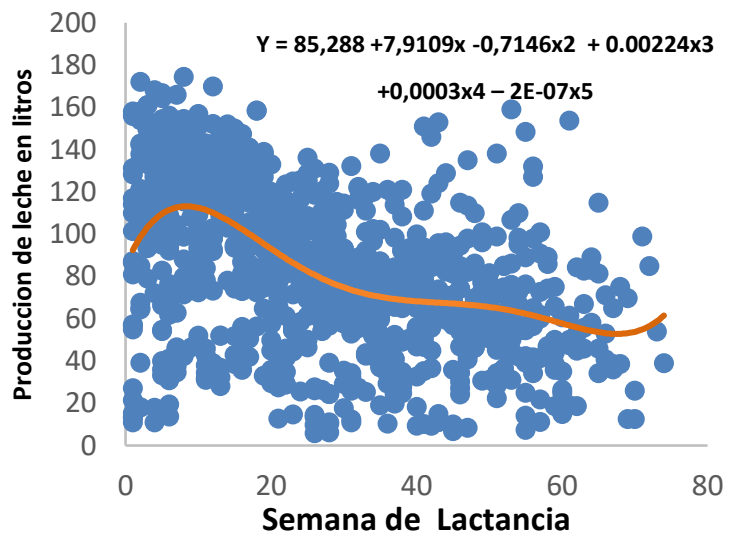

Elaborado: Segundo Shagñay. 2019.

La producción de leche generada en la tercera lactancia ajustada a 305 días de las vacas Holstein Friesian responde al modelo de quinto orden $\left(Y=85,288+7,9109 x-0,7146 x^{2}+0.00224 x^{3}\right.$ $\left.+0,0003 \mathrm{x}^{4}-2 \mathrm{E}-07 \mathrm{x}^{5}\right)$, lo que significa que por efecto del modelo en la producción cuando inicia la lactancia es de 85,288 litros/vaca/semana de lactancia y a medida que transcurre en el tiempo esta baja con relación a la 2da lactancia, esto se da en función a la disponibilidad de alimento, condiciones sanitarias, concluyéndose que la producción de leche está sujeto a la influencia de la fisiología de la glándula mamaria en la cual interviene factores hormonales que regulan la secreción de leche en los alveolos los cuales pueden afectar positiva y/o negativamente la misma.

\section{Modelación para la cuarta lactancia.}

Gráfico 1-4. Comportamiento Productivo en la cuarta lactancia de las vacas Holstein Friesan de la Estación Experimental Tunshi - ESPOCH. 


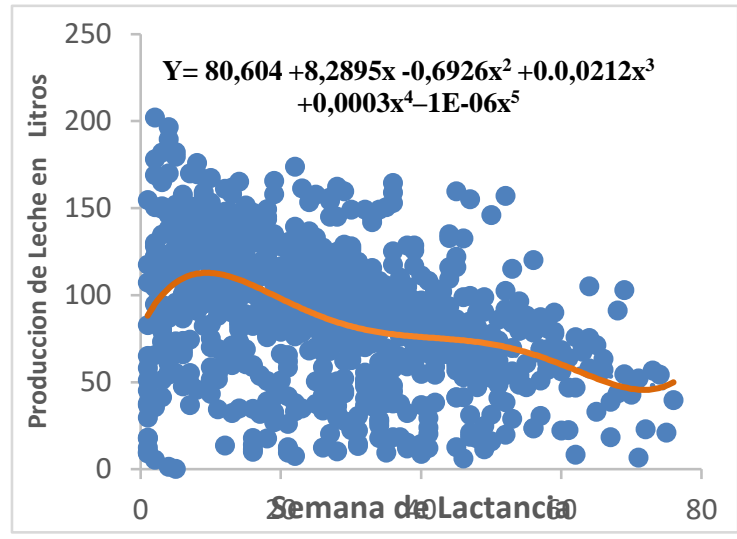

Elaborado: Segundo Shagñay. 2019.

La producción de leche generada en la cuarta lactancia ajustada a 305 días de las vacas Holstein Friesian responde al modelo de quinto orden $\left(Y=80,604+8,2895 \mathrm{x}-0,6926 \mathrm{x}^{2}+0.0,0212 \mathrm{x}^{3}\right.$ $\left.+0,0003 x^{4}-1 E-06 x^{5}\right)$, lo que significa que por efecto del modelo en la producción cuando inicia la lactancia es de 80,604 litros/vaca/semana, notándose una diferencia no significativa con relación a la 3ra lactancia, concluyéndose que a esta edad la vaca, ya desarrolla la glándula mamaria, estabiliza la producción láctea y se prepara para llegar al pico de su vida útil productiva donde mostrara su potencial genético.

\section{Modelación para la quinta lactancia.}

Gráfico 1-5. Comportamiento Productivo en la quinta lactancia de las vacas Holstein Friesan de la Estación Experimental Tunshi - ESPOCH

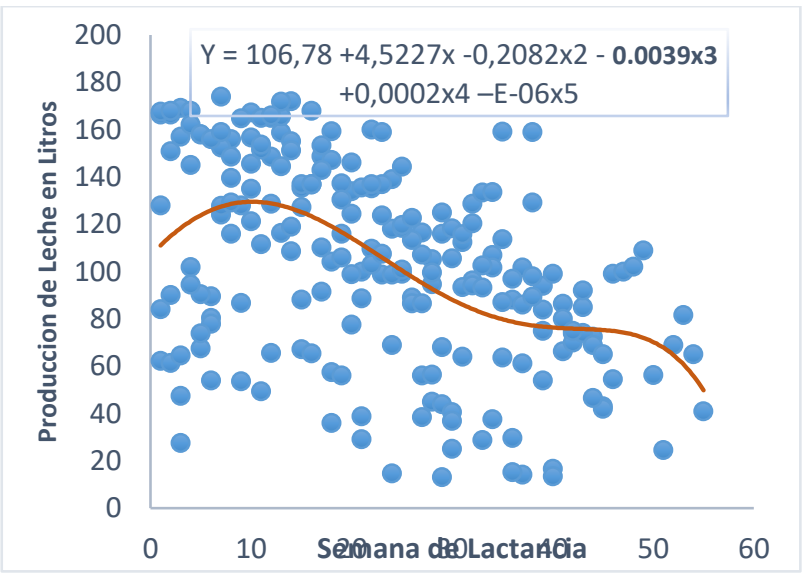

Elaborado: Segundo Shagñay. 2019.

La producción de leche generada en la quinta lactancia ajustada a 305 días de las vacas Holstein Friesian responde al modelo de quinto orden $\left(Y=106,78+4,5227 x-0,2082 x^{2}-0.0039 x^{3}\right.$ $\left.+0,0002 x^{4}-E-06 x^{5}\right)$, lo que significa que por efecto del modelo en la producción cuando inicia la lactancia es de 106,78 litros/vaca/semana, y a medida que esta va transcurriendo en el tiempo esta baja o sube de acuerdo a muchos factores que afecta la misma, se observa un incremento significativo de la producción al inicio de la lactancia con relación al anterior, esto se debe a que la vaca muestra su potencial genético, la secreción de leche esta en máximo nivel además 
influye el incremento de peso, el consumo de alimento, y principalmente el desarrollo de la glándula mamaria.

\section{Modelación para la sexta lactancia.}

Gráfico 1-6: Comportamiento Productivo en la sexta lactancia de las vacas Holstein Friesan de la Estación Experimental Tunshi - ESPOCH.

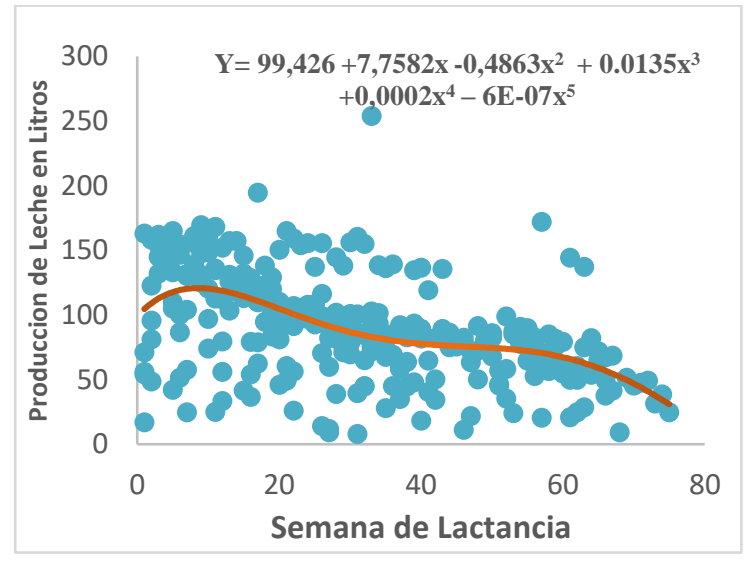

Elaborado: Segundo Shagñay. 2019.

La producción de leche generada en la sexta lactancia ajustada a 305 días de las vacas responden al modelo de quinto orden $\left(Y=99,426+7,7582 x-0,4863 x^{2}+0.0135 x^{3}+0,0002 x^{4}-6 E-07 x^{5}\right)$, lo que significa que por efecto del modelo la producción es de 99,426 litros/vaca/semana de lactancia; a medida que la vaca avaza en edad y lactancias la habilidad productiva baja con relación a la 5ta lactancia, en función de las condiciones de manejo de, alimentación y estado fisiológico de la vaca, la secreción de leche en la glándula mamaria comienza a descender, empieza la etapa de terminar su vida utlil reproductiva y productiva.

\section{Modelación para la séptima lactancia.}

Gráfico 1-7. Comportamiento Productivo en la séptima lactancia de las vacas Holstein Friesan de la Estación Experimental Tunshi - ESPOCH.

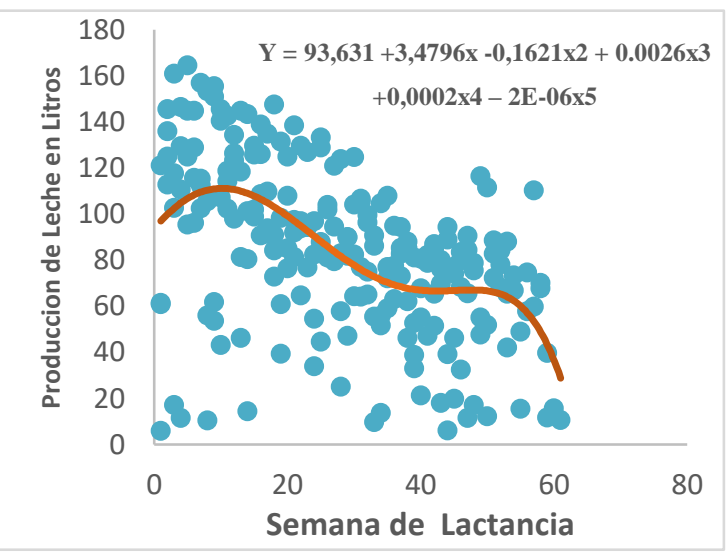

Elaborado: Segundo Shagñay. 2019. 
La producción de leche generada en la séptima lactancia ajustada a 305 días de las vacas Holstein Friesian responden al modelo de quinto orden $\left(\mathrm{Y}=93,631+3,4796 \mathrm{x}-0,1621 \mathrm{x}^{2}+\right.$ $\left.0.0026 \mathrm{x}^{3}+0,0002 \mathrm{x}^{4}-2 \mathrm{E}-06 \mathrm{x}^{5}\right)$, lo que significa que por efecto del modelo en la producción cuando inicia la lactancia es de 93,631 litros/vaca/semana, se observa que continua disminuyendo la producción de leche ,es to en función a las condiciones de, alimentación y estado fisiológico de la vaca, concluyéndose el cese de la producción llega cuando la vaca esta próxima al siguiente parto, factores de manejo, ambientales.

\section{Conclusiones}

- Los registros de producción diaria de las vacas son una herramienta muy útil para el análisis del comportamiento productivo durante cada lactancia.

- La producción de leche presenta un comportamiento ascendente relacionado con el desarrollo fisiológico del animal, la alimentación y los sistemas de manejo, en este estudio los resultados hacen notar que vacas de sexta lactancia expresan su potencial genético.

- El consumo de materia seca está directamente relacionado con el peso, edad, estado fisiológico, producción de leche y disponibilidad de alimento, lo que reportó el mayor consumo en la sexta lactancia debido a su producción.

- Los parámetros productivos evaluados en esta investigación responden a un modelo lineal de quinto orden cuyas ecuaciones describen correctamente el comportamiento de la producción lechera durante cada una de las lactancias

- Mediante las ecuaciones generadas en el presente estudio se pude predecir el comportamiento que tendrán las vacas bajos las condiciones actualmente manejadas

- Se cuenta con una herramienta útil para tomar decisiones de manejo técnico de la granja para mejorar futuras producciones.

\section{Referencias Bibliográficas}

Alvear, E. (2010), Caracterización Productiva y Reproductiva de la Hacienda "San Jorge" Para recomendar un Programa de Inseminación Artificial. (Tesis de Pregrado Inédita), ESPOCH-Riobamba - Ecuador.

Almeyda, J. M. 2014. Alimentación y manejo de vacunos lecheros. UNALM. Lima - Perú

Arango JP, Rivera B, Granobles J. (2000) Elaboración y validación de modelos de estimación de producción lechera en sistemas especializados. Trabajo de grado, Facultad de Ciencias Agropecuarias, Universidad de Caldas. En: Recopilación y Sistematización de los resultados de investigación de la Facultad de Ciencias Agropecuarias de la Universidad de Caldas. (CD ROM). Colombia. 120 p.

Dávalos, C. (2005) Caracterización de la Eficiencia Productiva y Reproductiva de dos hatos lecheros ubicados en la provincia de Chimborazo, durante el periodo 2002- 2003,(Tesisi de Pregrado Inedita), ESPOCH. R.bamba-Ecuador. 
Fabara, F. (2012). Estudio de Diferentes Niveles de Lasolacid en la Alimentación de vacas Holstein en la Hacienda Pucate. . (Tesis de Pregrado Inédita), ESPOCH-Riobamba Ecuador.

García, M. et al (2014). Criterios de información y predictivos para la selección de un modelo lineal mixto. Saberes. No 6:61 - 76. Sección Artículos

ICAR. 2008. International Committee for animal recording. International Agremment of recording practices, Guidelines approved by the General Assembly held in Niagara Falls. USA 18 June 2008.http://www.icar.org/Documents/Rules\%20and\%20regulations/Guidelines/ Guidelines_2009.pdf

(Instituto Nacional de Estadistica y Censo) 2015. Informe Tecnico Produccion Nacional No 3

Sagarpa, (2009). Secretaría de Agricultura,Ganadería, Desarrollo Rural, Pesca y Alimentación). 2004. Situación de la producción de leche bovina en México. Boletín informativo. México, D. F. p. 1-19.

Leon, J; Quiroz, J; Pleguezuelos, J; Martinez, E; Delgado, J. (2007). Curva de lactación para el numero de lactación en cabras murciano-granadinas. Arch. Zootec. 56(1): 641-646.

Torres, C. y Sosa, A . (2002). Manual Agropecuario. Editorial Printed. Bogotá - Colombia

Vélez, E. (2013). Factores de Origen Ambiental que afectan la Producción de leche en vacunos bajo pastoreo semi-intensivo. Universidad Nacional Mayor de San Marcos, Facultad de MedicinaVeterinaria. Peru- Cajamarca.

Velastegui, E. (2012). Administracion de GNRH y HCG post Inseminacion Artificial para incrementar la fertilidad en vacas Holstein Mestizas. . (Tesis de Pregrado Inédita), ESPOCH-Riobamba - Ecuador. 


\section{PARA CITAR EL ARTÍCULO INDEXADO.}

Shagñay Rea, S. M., Arias Alemán, L. S., \& Vaca Zambrano, S. E. (2020). La evaluación de las curvas de producción lechera de las vacas Holstein Friesian en la estación experimental Tunshi Espoch. Ciencia Digital, 4(1), 85-99. https://doi.org/10.33262/cienciadigital.v4i1.1070

\section{\Ciencia}

El artículo que se publica es de exclusiva responsabilidad de los autores y no necesariamente reflejan el pensamiento de la Revista Ciencia Digital.

El artículo queda en propiedad de la revista y, por tanto, su publicación parcial y/o total en otro medio tiene que ser autorizado por el director de la Revista Ciencia Digital.
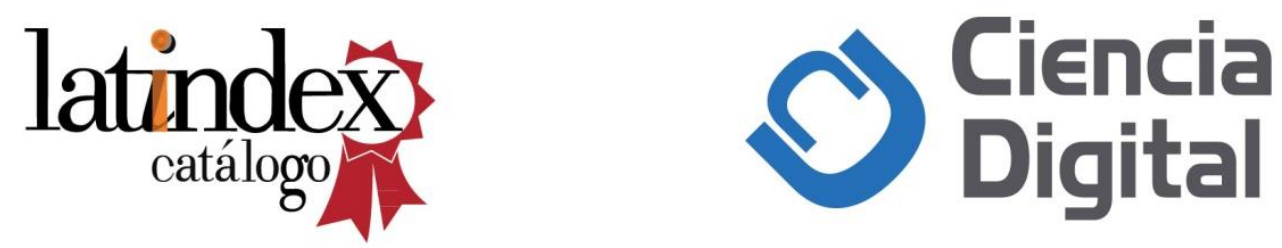